\title{
A Study on Novel Extensions for the $p$-adic Gamma and $p$-adic Beta Functions
}

\author{
Ugur Duran ${ }^{1, * \mathbb{D}}$ and Mehmet Acikgoz ${ }^{2}$ \\ 1 Department of the Basic Concepts of Engineering, Faculty of Engineering and Natural Sciences, \\ İskenderun Technical University, Hatay TR-31200, Turkey \\ 2 Department of Mathematics, Faculty of Science and Arts, University of Gaziantep, Gaziantep TR-27310, \\ Turkey; acikgoz@gantep.edu.tr \\ * Correspondence: ugur.duran@iste.edu.tr
}

Received: 23 April 2019; Accepted: 20 May 2019; Published: 21 May 2019

\begin{abstract}
In this paper, we introduce the $(\rho, q)$-analog of the $p$-adic factorial function. By utilizing some properties of $(\rho, q)$-numbers, we obtain several new and interesting identities and formulas. We then construct the $p$-adic $(\rho, q)$-gamma function by means of the mentioned factorial function. We investigate several properties and relationships belonging to the foregoing gamma function, some of which are given for the case $p=2$. We also derive more representations of the $p$-adic $(\rho, q)$-gamma function in general case. Moreover, we consider the $p$-adic $(\rho, q)$-Euler constant derived from the derivation of $p$-adic $(\rho, q)$-gamma function at $x=1$. Furthermore, we provide a limit representation of aforementioned Euler constant based on $(\rho, q)$-numbers. Finally, we consider $(\rho, q)$-extension of the $p$-adic beta function via the $p$-adic $(\rho, q)$-gamma function and we then investigate various formulas and identities.
\end{abstract}

Keywords: $p$-adic numbers; $p$-adic factorial function; $p$-adic gamma function; $p$-adic beta function; $p$-adic Euler constant; $(\rho, q)$-numbers

MSC: Primary 05A10, 05A30; Secondary 11B65, 11S80, 33B15

\section{Introduction}

The $p$-adic numbers are a counterintuitive arithmetic system, which were firstly introduced by Kummer in 1850. Then, the German mathematician Kurt Hensel (1861-1941) developed the $p$-adic numbers in a paper concerned with the development of algebraic numbers in power series in circa 1897 (cf. [1]). There are all kinds of numbers, such as natural, rational, real, complex, $p$-adic, and quantum numbers. The $p$-adic numbers are less well known than the others; however, these numbers play a main role in number theory and the related topics in mathematics. Since $p$-adic numbers have penetrated some mathematical areas, e.g., algebraic number theory, algebraic geometry, algebraic topology and analysis, they are now well-established in mathematical fields and are used also by physicists. In conjunction with the introduction of these numbers, some mathematicians and physicists started to investigate new scientific tools utilizing their useful and positive properties. Some effects of this new research have emerged in mathematics and physics, such as $p$-adic analysis, string theory, $p$-adic quantum mechanics, quantum field theory, representation theory, algebraic geometry, complex systems, dynamical systems, genetic codes and so on (cf. [1-22]). One of the most important tools of these investigations is $p$-adic gamma function, which was firstly described by Yasou Morita around 1975 (cf. [11]). Intense research activities in this area is principally motivated by its importance in $p$-adic analysis. Therefore, in the recent forty years, $p$-adic gamma function and its generalizations have been investigated and studied extensively by many mathematicians (cf. [1-13]). 
Here, we give some basic notations, definitions and properties belonging to the $p$-adic analysis which are taken from the books $[1,7,13]$.

Let $p \in\{2,3,5,7,11,13,17, \cdots\}$ be a prime number. For any nonzero integer $a$, let $\operatorname{ord}_{p} a$ be the highest power of $p$ that divides $a$, i.e., the greatest $m$ such that $a \equiv 0\left(\bmod p^{m}\right)$ where we used the notation $a \equiv b(\bmod c)$ meant $c$ divides $a-b$.

Note that $\operatorname{ord}_{p} 0=\infty$. The following properties hold true for $x=a b$ and $y=\frac{c}{d}$ :

$$
\operatorname{ord}_{p} x=\operatorname{ord}_{p} a+\operatorname{ord}_{p} b \text { and } \operatorname{ord}_{p} y=\operatorname{ord}_{p} c-\operatorname{ord}_{p} d .
$$

The $p$-adic absolute value (norm) of $x$ is given by

$$
|x|_{p}=\left\{\begin{array}{cc}
p^{-\operatorname{ord}_{p} x} & \text { for } x \neq 0 \\
0 & \text { for } x=0
\end{array}\right.
$$

The $p$-adic norm provides the so-called strong triangle inequality

$$
|x+y|_{p} \leqq \max \left\{|x|_{p},|y|_{p}\right\}
$$

which is also known as non-Archimedean norm.

Now, we provide some basic notations: $\mathbb{N}=\{1,2,3, \cdots\}$ denotes the set of all natural numbers, $\mathbb{Z}=\{\cdots,-1,0,1, \cdots\}$ denotes the ring of all integers, $\mathbb{Q}=\left\{\frac{a}{b} \mid a, b \in \mathbb{Z}, b \neq 0\right\}$ denotes the field of all rational numbers, $\mathbb{C}$ denotes the field of all complex numbers, $\mathbb{Q}_{p}=$ $\left\{x=\sum_{n=-k}^{\infty} a_{n} p^{n}: 0 \leqq a_{i} \leqq p-1\right\}$ denotes the field of all $p$-adic numbers, $\mathbb{Z}_{p}=\left\{x \in \mathbb{Q}_{p}:|x|_{p} \leqq 1\right\}$ denotes the ring of all $p$-adic integers and $\mathbb{C}_{p}$ denotes the completion of the algebraic closure of $\mathbb{Q}_{p}$. Let $\mathbb{N}_{0}=\mathbb{N} \cup\{0\}$.

For more information about $p$-adic analysis, see, e.g., [1-22].

The notations $\rho$ and $q$ can be variously considered as indeterminates, complex numbers $\rho$ and $q \in$ $\mathbb{C}$ with $0<|q|<|\rho| \leqq 1$, or $p$-adic numbers $\rho$ and $q \in \mathbb{C}_{p}$ with $|\rho-1|_{p}<p^{-\frac{1}{p-1}}$ and $|q-1|_{p}<p^{-\frac{1}{p-1}}$ so that $\rho^{x}=\exp (x \log \rho)$ and $q^{x}=\exp (x \log q)$ for $|x|_{p} \leqq 1$.

The classical gamma function is firstly introduced by Leonard Euler (1707-1783) as

$$
\Gamma(x)=\int_{0}^{1}(-\log t)^{x-1} d t \quad(x>0) .
$$

In 1964, the common form of the gamma function was given by Artin [23] with appropriate variable change:

$$
\Gamma(x)=\int_{0}^{\infty} t^{x-1} e^{-t} d t \quad(x>0) .
$$

The classical gamma function is closely related with the factorial function $n !$ as $\Gamma(n+1)=n$ ! for $n \in \mathbb{N}$.

By inspiring the beautiful and interesting relation between gamma function and factorial function above, the $p$-adic gamma function is also introduced by means of the $p$-adic factorial function $(n !)_{p}$ as follows

$$
\Gamma_{p}(x)=\lim _{n \rightarrow x}(-1)^{n}(n !)_{p},
$$

where the factorial function $(n !)_{p}$ in $\mathbb{Q}_{p}$ is defined by

$$
(n !)_{p}=\prod_{\substack{j<n \\(p, j)=1}} j
$$

for $x \in \mathbb{Z}_{p}$, where $n$ approaches $x$ through positive integers. For detailed statement of these issue, see $[1,4,5,7,11,13]$. 
The $q$-extension of the $p$-adic gamma function is defined as follows (see [12])

$$
\Gamma_{p, q}(x)=\lim _{n \rightarrow x}(-1)^{n} \prod_{\substack{j<n \\(p, j)=1}}[j]_{q} \text { where }[j]_{q}=\frac{1-q^{j}}{1-q}
$$

These functions have been studied and investigated by many mathematicians, see $[3-9,11,12]$.

The $(\rho, q)$-numbers are defined by

$$
[n]_{\rho, q}:=\frac{\rho^{n}-q^{n}}{\rho-q}
$$

which reduce to the $q$-numbers when $\rho=1$ as $[n]_{1, q} \rightarrow[n]_{q}$.

It is clear that $[n]_{\rho, q}=\rho^{n-1}[n]_{q / \rho}$, which means that $q$-numbers and $(\rho, q)$-numbers are different, that is, $(\rho, q)$-numbers cannot be obtained just by substituting $q$ by $q / \rho$ in the definition of $q$-numbers (see $[15-20,24,25]$ for details). However, when $\rho=1, q$-numbers become a special case of $(\rho, q)$-numbers, as shown above.

In conjunction with the introduction of these $(\rho, q)$-numbers (see [24]), $(\rho, q)$-calculus has been investigated and studied extensively by many mathematicians and also physicists since 1991 . For example, Araci et al. [15] introduced an analog of Haar distribution based on $(\rho, q)$-numbers. By means of this distribution, they derived $(\rho, q)$-analog of Volkenborn integral ( $p$-adic integral) and obtained some properties. Then, they constructed $(\rho, q)$-Bernoulli polynomials arising from $(\rho, q)$-Volkenborn integral. Aral et al. [16] defined a $(\rho, q)$-analog of Gamma function and, as an application, they proposed $(\rho, q)$-Szasz-Durrmeyer operators, estimated moments and established some direct results. Chakrabarti et al. [24] investigated the necessary elements of the $(\rho, q)$-calculus involving $(\rho, q)$-exponential, $(\rho, q)$-integration, and the $(\rho, q)$-differentiation. Duran et al. [17] considered a generalization of the fermionic $p$-adic measure based on $(\rho, q)$-integers and set the corresponding integral to this measure. They also defined Carlitz-type $(\rho, q)$-Euler polynomials and numbers in terms of this corresponding integral and acquired some of their identities and properties. Milovanovic et al. [25] provided a novel extension of beta functions based on $(\rho, q)$-numbers and committed the integral modification of the generalized Bernstein polynomials. Sadjang [18] introduced new generalizations of the gamma and the beta functions and investigated their properties. Sadjang [19] investigated some properties of the $(\rho, q)$-derivative and the $(\rho, q)$-integration and provided two appropriate polynomial bases for the $(\rho, q)$-derivative, and then he obtained various properties of these bases. As an application, he gave two $(\rho, q)$-Taylor formulas for polynomials. Furthermore, he gave the fundamental theorem of $(\rho, q)$-calculus and proved the formula of $(\rho, q)$-integration by part. Sahai et al. [20] developed the connection between $(\rho, q)$-analog of special functions and representations of certain two parameter quantum algebras.

The paper is organized as follows. Section 1 , the Introduction, provides the required information, notations, definitions and motivation. In Section 2, we are interested in constructing the $p$-adic $(\rho, q)$-gamma function $\Gamma_{p}^{[\rho, q]}(x)$ by means of $p$-adic $(\rho, q)$-factorial function $(x !)_{p}^{[\rho, q]}$. We investigate some properties and relationships of the mentioned gamma function. In Section 3 , the $p$-adic $(\rho, q)$-Euler constant is derived from the derivation of $p$-adic $(\rho, q)$-gamma function at $x=1$ and limit representation of this constant are shown. In Section 3, we also examine the results derived in this paper and give some further remarks of our results. Section 4 provides the $(\rho, q)$-extension of the $p$-adic beta function via the $p$-adic $(\rho, q)$-gamma function and includes multifarious formulas and identities.

\section{The $p$-adic $(\rho, q)$-Gamma Function}

This section provides a new definition of $p$-adic $(\rho, q)$-gamma function and gives some properties, identities and relations for the mentioned gamma function. 
We firstly introduce $(\rho, q)$-extension of the $p$-adic factorial function as follows.

Definition 1. Let $\rho$ and $q \in \mathbb{C}_{p}$ with $|\rho-1|_{p}<1$ and $|q-1|_{p}<1, \rho \neq 1$ and $q \neq 1$. We introduce the p-adic $(\rho, q)$-factorial function $(x !)_{p}^{[\rho, q]}$ in $\mathbb{Q}_{p}$ as

$$
(x !)_{p}^{[\rho, q]}=\lim _{n \rightarrow x} \prod_{\substack{j<n \\(p, j)=1}} \frac{\rho^{j}-q^{j}}{\rho-q}=\lim _{n \rightarrow x} \prod_{\substack{j<n \\(p, j)=1}}[j]_{\rho, q}
$$

for $x \in \mathbb{Z}_{p}$, where $n$ approaches $x$ through positive integers.

Note that, for $n \in \mathbb{N}$, the $p$-adic $(\rho, q)$-factorial function can be written as

$$
(n !)_{p}^{[\rho, q]}=\prod_{\substack{j<n \\(p, j)=1}}[j]_{\rho, q} .
$$

Proposition 1. For $n \in \mathbb{N}$, we have

$$
(1 !)_{p}^{[\rho, q]}=1,(2 !)_{p}^{[\rho, q]}=1 \text { and }\left|(n !)_{p}^{[\rho, q]}\right|_{p}=1 .
$$

Example 1. We provide some examples of the foregoing function:

\begin{tabular}{ccc}
\hline$(3 !)_{2}^{[\rho, q]}=1$ & $(3 !)_{3}^{[\rho, q]}=[2]_{\rho, q}$ & $(3 !)_{5}^{[\rho, q]}=[2]_{\rho, q}$ \\
\hline$(6 !)_{2}^{[\rho, q]}=[3]_{\rho, q}[5]_{\rho, q}$ & $(6 !)_{3}^{[\rho, q]}=[2]_{\rho, q}[4]_{\rho, q}[5]_{\rho, q}$ & $(6 !)_{5}^{[\rho, q]}=[2]_{\rho, q}[3]_{\rho, q}[4]_{\rho, q}$ \\
$(7 !)_{2}^{[\rho, q]}=[3]_{\rho, q}[5]_{\rho, q}$ & $(7 !)_{3}^{[\rho, q]}=[2]_{\rho, q}[4]_{\rho, q}[5]_{\rho, q}$ & $(7 !)_{5}^{[\rho, q]}=[2]_{\rho, q}[3]_{\rho, q}[4]_{\rho, q}[6]_{\rho, q}$ \\
\hline
\end{tabular}

By Equation (5), we note that

$$
[n+m]_{\rho, q}=\rho^{n}[m]_{\rho, q}+q^{m}[n]_{\rho, q}=\rho^{m}[n]_{\rho, q}+q^{n}[m]_{\rho, q} .
$$

Using the addition property in Equation (8) of the $(\rho, q)$-integers, we give the following theorem.

Theorem 1. For $n, m \in \mathbb{N}$, we have

$$
((n+m) !)_{p}^{[\rho, q]}=(n !)_{p}^{[\rho, q]} \cdot\left\{\begin{array}{l}
\left(\rho^{n}(m !)_{p}^{[\rho, q]}+[n]_{\rho, q} q^{\left(\begin{array}{c}
m-1 \\
2
\end{array}\right)-p\left(1+2+\ldots+\left\lfloor\frac{m-1}{p}\right]\right)}\right) \text { if } d=0 \\
\rho^{n} \prod_{\substack{j<m \\
(p, d+j)=1}}[j]_{\rho, q}+[n]_{\rho, q} \prod_{\substack{j<m \\
(p, d+j)=1}} q^{j} \quad \text { if } d \in A
\end{array},\right.
$$

where $n=p k+d$ and $A=\{1,2, \ldots, p-1\}$ and $\lfloor\cdot\rfloor$ is the greatest integer function. 
Proof. In view of Equations (6) and (8), we get

$$
\begin{aligned}
& ((n+m) !)_{p}^{[\rho, q]}=\prod_{\substack{j<n+m \\
(p, j)=1}}[j]_{\rho, q}=\prod_{\substack{j<n \\
(p, j)=1}}[j]_{\rho, q} \prod_{\substack{j<m \\
(p, n+j)=1}}[n+j]_{\rho, q} \\
& =\prod_{\substack{j<n \\
(p, j)=1}}[j]_{\rho, q} \prod_{\substack{j<m \\
(p, n+j)=1}}\left(\rho^{n}[j]_{\rho, q}+q^{j}[n]_{\rho, q}\right) \\
& =(n !)_{p}^{[\rho, q]}\left(\rho^{n} \prod_{\substack{j<m \\
(p, n+j)=1}}[j]_{\rho, q}+[n]_{\rho, q} \prod_{\substack{j<m \\
(p, n+j)=1}} q^{j}\right)
\end{aligned}
$$

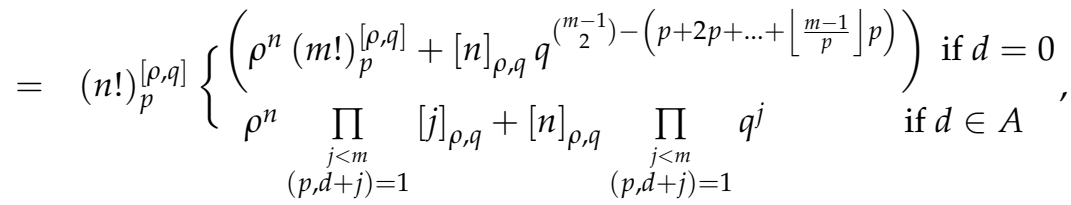

where $n=p k+d$ and $A=\{1,2, \ldots, p-1\}$. Thus, we attain the asserted result in Equation (9).

We give the following interesting result.

Theorem 2. For $m \in \mathbb{N}$, we have

$$
\left(\varphi_{p}(m) !\right)_{p}^{[\rho, q]}=\left(a_{0} !\right)_{p}^{[\rho, q]} \prod_{t=1}^{m} \prod_{\substack{j<a_{p} p^{t} \\(p, j)=1}}\left[\varphi_{p}(t-1)+j\right]_{\rho, q^{\prime}},
$$

where $\varphi_{p}(m)=a_{0}+a_{1} p+a_{2} p^{2}+\cdots+a_{m} p^{m}$ with $a_{0}, a_{1}, \ldots a_{m} \in\{1,2, \ldots, p-1\}$.

Proof. Indeed,

$$
\begin{aligned}
\left(\varphi_{p}(m) !\right)_{p}^{[\rho, q]}= & \left(\left(\varphi_{p}(m-1)\right) !\right)_{p}^{[\rho, q]} \prod_{\substack{j<a_{p} p^{m} \\
(p, j)=1}}\left[\varphi_{p}(m-1)+j\right]_{\rho, q} \\
= & \left(\left(\varphi_{p}(m-2)\right) !\right)_{p}^{[\rho, q]} \prod_{\substack{j<a_{m-1} p^{m-1} \\
(p, j)=1}}\left[\varphi_{p}(m-2)+j\right]_{\rho, q} \prod_{\substack{j<a_{m} p^{m} \\
(p, j)=1}}\left[\varphi_{p}(m-1)+j\right]_{\rho, q} \\
& \vdots \\
= & \left(a_{0} !\right)_{p}^{[\rho, q]} \prod_{t=1}^{m} \prod_{\substack{j<a_{p} p^{t} \\
(p, j)=1}}\left[\varphi_{p}(t-1)+j\right]_{\rho, q^{\prime}}
\end{aligned}
$$

which completes the proof of this theorem.

The following definition is new and plays an important role in deriving the main results of this paper. Now, we are ready to state the following Definition 2.

Definition 2. Let $\rho$ and $q \in \mathbb{C}_{p}$ with $|\rho-1|_{p}<1$ and $|q-1|_{p}<1, \rho \neq 1$ and $q \neq 1$. We define the $p$-adic $(\rho, q)$-gamma function as follows

$$
\Gamma_{p}^{[\rho, q]}(x)=\lim _{n \rightarrow x}(-1)^{n} \prod_{\substack{j<n \\(p, j)=1}} \frac{\rho^{j}-q^{j}}{\rho-q}=\lim _{n \rightarrow x}(-1)^{n} \prod_{\substack{j<n \\(p, j)=1}}[j]_{\rho, q}
$$


for $x \in \mathbb{Z}_{p}$, where $n$ approaches $x$ through positive integers.

Note that for $n \in \mathbb{N}$, the $p$-adic $(\rho, q)$-gamma function can be written as

$$
\Gamma_{p}^{[\rho, q]}(n)=(-1)^{n} \prod_{\substack{j<n \\(p, j)=1}}[j]_{\rho, q} .
$$

Example 2. We give some examples of the aforementioned function:

\begin{tabular}{ccc}
\hline$\Gamma_{2}^{[\rho, q]}(3)=-1$ & $\Gamma_{3}^{[\rho, q]}(3)=-[2]_{\rho, q}$ & $\Gamma_{5}^{[\rho, q]}(3)=-[2]_{\rho, q}$ \\
\hline$\Gamma_{2}^{[\rho, q]}(6)=[3]_{\rho, q}[5]_{\rho, q}$ & $\Gamma_{3}^{[\rho, q]}(6)=[2]_{\rho, q}[4]_{\rho, q}[5]_{\rho, q}$ & $\Gamma_{5}^{[\rho, q]}(6)=[2]_{\rho, q}[3]_{\rho, q}[4]_{\rho, q}$ \\
$\Gamma_{2}^{[\rho, q]}(7)=-[3]_{\rho, q}[5]_{\rho, q}$ & $\Gamma_{3}^{[\rho, q]}(7)=-[2]_{\rho, q}[4]_{\rho, q}[5]_{\rho, q}$ & $\Gamma_{5}^{[\rho, q]}(7)=-[2]_{\rho, q}[3]_{\rho, q}[4]_{\rho, q}[6]_{\rho, q}$ \\
\hline
\end{tabular}

Remark 1. Upon setting $\rho=1$ in Definition $2, p$-adic $(\rho, q)$-gamma function reduces to the $p$-adic $q$-gamma function in Equation (4).

Remark 2. When $q \rightarrow \rho=1$ in Definition 2, Equation (11) yields to the $p$-adic gamma function in Equation (2).

We now investigate some properties and relations of the aforementioned function.

Lemma 1. For $n \in \mathbb{N}$, we have

$$
\Gamma_{p}^{[\rho, q]}(0)=1, \Gamma_{p}^{[\rho, q]}(1)=-1, \Gamma_{p}^{[\rho, q]}(2)=1 \text { and }\left|\Gamma_{p}^{[\rho, q]}(n)\right|_{p}=1 .
$$

Proof. The proof of this lemma just follows from the Definition 2. Thus, we omit the proof.

Taking into account Theorem 1, we obtain the following relation.

Corollary 1. For $n, m \in \mathbb{N}$, we have

$$
\Gamma_{p}^{[\rho, q]}(n+m)=(-1)^{n+m} \Gamma_{p}^{[\rho, q]}(n) \cdot\left\{\begin{array}{c}
\left(\rho^{n} \Gamma_{p}^{[\rho, q]}(m)+[n]_{\rho, q} q^{(m-1)}-p\left(1+2+\ldots+\left\lfloor\frac{m-1}{p}\right\rfloor\right)\right) \\
\rho^{n} \prod_{\substack{j<m \\
(p, d+j)=1}}[j]_{\rho, q}+[n]_{\rho, q} \prod_{\substack{j<m \\
(p, d+j)=1}} q^{j} \quad \text { if } d=0
\end{array},\right.
$$

where $n=p k+d$ and $A=\{1,2, \ldots, p-1\}$ and $\lfloor\cdot\rfloor$ is the greatest integer function.

Considering that Theorem 2, we have the following identity.

Corollary 2. For $m \in \mathbb{N}_{0}$, we have

$$
\Gamma_{p}^{[\rho, q]}\left(\varphi_{p}(m)\right)=(-1)^{\varphi_{p}(m)}\left(a_{0} !\right)_{p}^{[\rho, q]} \prod_{t=1}^{m} \prod_{\substack{j<a_{p} p^{t} \\(p, j)=1}}\left[\varphi_{p}(t-1)+j\right]_{\rho, q^{\prime}}
$$

where $\varphi_{p}(m)=a_{0}+a_{1} p+a_{2} p^{2}+\cdots+a_{m} p^{m}$ with $a_{0}, a_{1}, \ldots a_{m} \in\{1,2, \ldots, p-1\}$.

Here is a recurrence relation for $\Gamma_{p}^{[\rho, q]}(n)$ by the following theorem. 
Theorem 3. The following recurrence formula holds true for all $x \in \mathbb{Z}_{p}$ :

$$
\Gamma_{p}^{[\rho, q]}(x+1)=\epsilon_{p}^{[\rho, q]}(x) \Gamma_{p}^{[\rho, q]}(x),
$$

where

$$
\epsilon_{p}^{[\rho, q]}(x)=\left\{\begin{array}{cc}
-[x]_{\rho, q} & \text { if }|x|_{p}=1 \\
-1 & \text { if }|x|_{p}<1
\end{array}\right.
$$

Proof. Using Definition 2 and Equation (1), we easily get

$$
\Gamma_{p}^{[\rho, q]}(x+1)=\lim _{n \rightarrow x}(-1)^{n+1} \prod_{\substack{j<n+1 \\
(p, j)=1}}[j]_{\rho, q}=\left(\lim _{n \rightarrow x}(-1)^{n} \prod_{\substack{j<n \\
(p, j)=1}}[j]_{\rho, q}\right) \cdot\left\{\begin{array}{ccc}
-[x]_{\rho, q} & \text { if }|x|_{p}=1 \\
-1 & \text { if } & |x|_{p}<1
\end{array}\right.
$$

which gives the desired result in Equation (12).

The result obtained in the Theorem 3 seems to be the $p$-adic $(\rho, q)$-analog of the well known result for classical gamma function $\Gamma(x+1)=x \Gamma(x)$ for $x>0$.

We now give an explicit formula for $\Gamma_{p}^{[\rho, q]}(n)$ as follows.

Theorem 4. The following recurrence formula holds true for all $n \in \mathbb{N}$ :

$$
\Gamma_{p}^{[\rho, q]}(n+1)=(-1)^{n+1} \frac{[n]_{\rho, q} !}{[p]_{\rho, q}^{\left\lfloor\frac{n}{p}\right\rfloor}\left[\left\lfloor\frac{n}{p}\right\rfloor\right]_{\rho^{p}, q^{p}} !},
$$

where $\lfloor\cdot\rfloor$ is the greatest integer function.

Proof. From Definition 2, we observe that

$$
\begin{aligned}
\Gamma_{p}^{[\rho, q]}(n+1) & =(-1)^{n+1} \prod_{\substack{j<n \\
(p, j)=1}}[j]_{\rho, q} \\
& =(-1)^{n+1} \frac{[1]_{\rho, q}[2]_{\rho, q} \cdots[n]_{\rho, q}}{[p]_{\rho, q}[2 p]_{\rho, q} \cdots\left[\left\lfloor\frac{n}{p}\right\rfloor p\right]_{\rho, q}} .
\end{aligned}
$$

Using the product rule $[k p]_{\rho, q}=[k]_{\rho^{p}, q^{p}}[p]_{\rho, q}$ for $(\rho, q)$-numbers, we acquire

$$
\Gamma_{p}^{[\rho, q]}(n+1)=(-1)^{n+1} \frac{[n]_{\rho, q} !}{[p]_{\rho, q}^{\left\lfloor\frac{n}{p}\right\rfloor}[1]_{\rho^{p}, q^{p}}[2]_{\rho^{p}, q^{p}} \cdots\left[\left\lfloor\frac{n}{p}\right\rfloor\right]_{\rho^{p}, q^{p}}},
$$

which yields to the asserted result in Equation (14).

Particularly, we derive the following result.

Corollary 3. We have

$$
\Gamma_{p}^{[\rho, q]}\left(p^{n}\right)=(-1)^{p} \frac{\left[p^{n}-1\right]_{\rho, q} !}{[p]_{\rho, q}^{p^{n-1}-1}\left[p^{n-1}-1\right]_{\rho^{p}, q^{p}} !} .
$$

Here are two relations for $\Gamma_{p}^{[\rho, q]}(x)$ and the latter provides a representation of $(\rho, q)$-factorial function associated with $p$-adic $(\rho, q)$-gamma function. 
Theorem 5. For $n \in \mathbb{N}$, let $m_{n}$ be the sum of digits of $n=\sum_{j=0}^{m} a_{j} p^{j}\left(a_{m} \neq 0\right)$ in base $p$. We then derive

$$
\left[\left\lfloor\frac{n}{p^{m}}\right\rfloor\right]_{\rho, q} !=(-1)^{n+1-m}\left(-[p]_{\rho, q}\right)^{\left(n-m_{n}\right) /(p-1)} \prod_{j=0}^{m-1} \frac{\left[\left\lfloor\frac{n}{p^{j+1}}\right\rfloor\right]_{\rho^{p}, q^{p}} !}{\left\lfloor\left\lfloor\frac{n}{p^{j}}\right\rfloor\right]_{\rho, q} !} \prod_{i=0}^{m} \Gamma_{p}^{[\rho, q]}\left(\left\lfloor\frac{n}{p^{i}}\right\rfloor+1\right)
$$

and

$$
[n]_{\rho, q} !=(-1)^{n+1-m}\left(-[p]_{\rho, q}\right)^{\left(n-m_{n}\right) /(p-1)}\left[\left\lfloor\frac{n}{p}\right\rfloor\right]_{\rho^{p}, q^{p}} ! \prod_{j=1}^{m} \frac{\left[\left\lfloor\frac{n}{p^{j+1}}\right\rfloor\right]_{\rho^{p}, q^{p}} !}{\left\lfloor\left\lfloor\frac{n}{p^{j}}\right\rfloor\right]_{\rho, q} !} \prod_{i=0}^{m} \Gamma_{p}^{[\rho, q]}\left(\left\lfloor\frac{n}{p^{i}}\right\rfloor+1\right) .
$$

Proof. By Equation (14), we have

$$
[n]_{\rho, q} !=(-1)^{n+1}[p]_{\rho, q}^{\left\lfloor\frac{n}{p}\right\rfloor}\left[\left\lfloor\frac{n}{p}\right\rfloor\right]_{\rho^{p}, q^{p}} ! \Gamma_{p}^{[\rho, q]}(n+1) .
$$

Then, if we put $\left\lfloor\frac{n}{p^{j}}\right\rfloor$ where $j$ lies in $\{0,1, \cdots, m\}$ instead of $n$, respectively, we observe that

$$
\begin{gathered}
\left\lfloor\left\lfloor\frac{n}{p^{0}}\right\rfloor\right]_{\rho, q} !=(-1)^{\left\lfloor\frac{n}{p^{0}}\right\rfloor+1}[p]_{\rho, q}^{\left\lfloor\frac{n}{p^{1}}\right\rfloor}\left[\left\lfloor\frac{n}{p^{1}}\right\rfloor\right]_{\rho^{p}, q^{p}} ! \Gamma_{p}^{[\rho, q]}\left(\left\lfloor\frac{n}{p^{0}}\right\rfloor+1\right) \\
{\left[\left\lfloor\frac{n}{p^{1}}\right\rfloor\right]_{\rho, q}=(-1)^{\left\lfloor\frac{n}{p^{1}}\right\rfloor+1}[p]_{\rho, q}^{\left.\frac{n}{p^{2}}\right\rfloor}\left\lfloor\left\lfloor\frac{n}{p^{2}}\right\rfloor\right]_{\rho^{p}, q^{p}} ! \Gamma_{p}^{[\rho, q]}\left(\left\lfloor\frac{n}{p^{1}}\right\rfloor+1\right)} \\
\vdots \vdots \\
{\left[\left\lfloor\frac{n}{p^{m}}\right\rfloor\right]_{\rho, q} !=(-1)^{\left\lfloor\frac{n}{p^{m}}\right\rfloor+1}[p]_{\rho, q}^{\left\lfloor\frac{n}{p^{m+1}}\right\rfloor}\left[\left\lfloor\frac{n}{p^{m+1}}\right\rfloor\right]_{\rho^{p}, q^{p}} ! \Gamma_{p}^{[\rho, q]}\left(\left\lfloor\frac{n}{p^{m}}\right\rfloor+1\right) .}
\end{gathered}
$$

Multiplying the both sides above, one can acquire with ease that

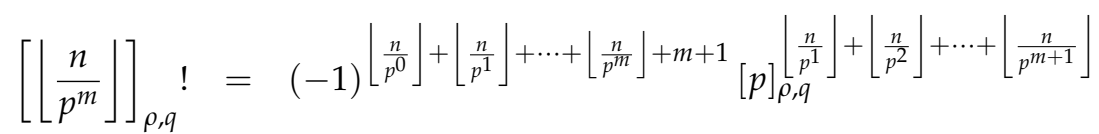

$$
\begin{aligned}
& \cdot\left[\left\lfloor\frac{n}{p^{m+1}}\right\rfloor\right]_{\rho^{p}, q^{p}} ! \prod_{j=0}^{m-1} \frac{\left[\left\lfloor\frac{n}{p^{j+1}}\right\rfloor\right]_{\rho^{p}, q^{p}} !}{\left[\left\lfloor\frac{n}{p^{j}}\right\rfloor\right]_{\rho, q} !} \prod_{i=0}^{m} \Gamma_{p}^{[\rho, q]}\left(\left\lfloor\frac{n}{p^{i}}\right\rfloor+1\right) \\
& =(-1)^{\left(n-m_{n}\right) /(p-1)}(-1)^{n+1-m}[p]_{\rho, q}^{\left(n-m_{n}\right)(p-1)}\left[\left\lfloor\frac{n}{p^{m+1}}\right\rfloor\right]_{\rho^{p}, q^{p}} \text { ! } \\
& \cdot \prod_{j=0}^{m-1} \frac{\left[\left\lfloor\frac{n}{p^{j+1}}\right\rfloor\right]_{\rho^{p}, q^{p}} !}{\left[\left\lfloor\frac{n}{p^{j}}\right\rfloor\right]_{\rho, q} !} \prod_{i=0}^{m} \Gamma_{p}^{[\rho, q]}\left(\left\lfloor\frac{n}{p^{i}}\right\rfloor+1\right) .
\end{aligned}
$$


Thus, we get the asserted result in Equation (16):

$$
\begin{aligned}
{\left[\left\lfloor\frac{n}{p^{m}}\right\rfloor\right]_{\rho, q} !=} & (-1)^{n+1-m}\left(-[p]_{\rho, q}\right)^{\left(n-m_{n}\right)(p-1)} \\
& \cdot \prod_{j=0}^{m-1} \frac{\left\lfloor\left\lfloor\frac{n}{p^{j+1}}\right\rfloor\right]_{\rho^{p}, q^{p}} !}{\left[\left\lfloor\frac{n}{p^{j}}\right\rfloor\right]_{\rho, q} !} \prod_{i=0}^{m} \Gamma_{p}^{[\rho, q]}\left(\left\lfloor\frac{n}{p^{i}}\right\rfloor+1\right) .
\end{aligned}
$$

In addition, from the applications above,

$$
\begin{aligned}
{[n]_{\rho, q} !=} & (-1)^{\left\lfloor\frac{n}{p^{0}}\right\rfloor+\left\lfloor\frac{n}{p^{1}}\right\rfloor+\cdots+\left\lfloor\frac{n}{p^{m}}\right\rfloor+m+1}[p]_{\rho, q}\left\lfloor\frac{n}{p^{1}}\right\rfloor+\left\lfloor\frac{n}{p^{2}}\right\rfloor+\cdots+\left\lfloor\frac{n}{p^{m+1}}\right\rfloor \\
& \cdot\left[\left\lfloor\frac{n}{p}\right\rfloor\right]_{\rho^{p}, q^{p}} ! \prod_{j=1}^{m} \frac{\left\lfloor\left\lfloor\frac{n}{p^{j+1}}\right\rfloor\right]_{\rho^{p}, q^{p}} !}{\left[\left\lfloor\frac{n}{p^{j}}\right\rfloor\right]_{\rho, q} !} \prod_{i=0}^{m} \Gamma_{p}^{[\rho, q]}\left(\left\lfloor\frac{n}{p^{i}}\right\rfloor+1\right) \\
= & (-1)^{\left(n-m_{n}\right)(p-1)}(-1)^{n+1-m}[p]_{\rho, q}^{\left(n-m_{n}\right)(p-1)}\left[\left\lfloor\frac{n}{p}\right\rfloor\right]_{\rho^{p}, q^{p}} ! \\
& \cdot \prod_{j=1}^{m} \frac{\left[\left\lfloor\frac{n}{p^{j+1}}\right\rfloor\right]_{\rho^{p}, q^{p}} !}{\left[\left\lfloor\frac{n}{p^{j}}\right\rfloor\right]_{\rho, q} !} \prod_{i=0}^{m} \Gamma_{p}^{[\rho, q]}\left(\left\lfloor\frac{n}{p^{i}}\right\rfloor+1\right) .
\end{aligned}
$$

Thus, we obtain Equation (17):

$$
\begin{aligned}
{[n]_{\rho, q} !=} & (-1)^{n+1-m}\left(-[p]_{\rho, q}\right)^{\left(n-m_{n}\right) /(p-1)}\left[\left\lfloor\frac{n}{p}\right\rfloor\right]_{\rho^{p}, q^{p}} ! \\
& \cdot \prod_{j=1}^{m} \frac{\left[\left\lfloor\frac{n}{p^{j+1}}\right\rfloor\right]_{\rho^{p}, q^{p}} !}{\left[\left\lfloor\frac{n}{p^{j}}\right\rfloor\right]_{\rho, q} !} \prod_{i=0}^{m} \Gamma_{p}^{[\rho, q]}\left(\left\lfloor\frac{n}{p^{i}}\right\rfloor+1\right) .
\end{aligned}
$$

We give the following theorem.

Theorem 6. The following relation holds true for any prime $p$ and $n \in \mathbb{N}$ :

$$
\begin{aligned}
{\left[p^{n}-1\right]_{\rho, q} !=} & (-1)^{p}\left(-[p]_{\rho, q}\right)^{\left(p^{n}-1\right) /(p-1)}[p]_{\rho, q}^{-n}\left[p^{n-1}-1\right]_{\rho^{p}, q^{p}} ! \\
& \cdot \prod_{j=0}^{n-2} \frac{\left[p^{j}-1\right]_{\rho^{p}, q^{p}}}{\left[p^{j+1}-1\right]_{\rho, q}} \prod_{j=0}^{n} \Gamma_{p}^{[\rho, q]}\left(p^{j}\right) .
\end{aligned}
$$

Proof. In view of Equation (15), we have

$$
\left[p^{k}-1\right]_{\rho, q} !=(-1)^{p} \Gamma_{p}^{[\rho, q]}\left(p^{k}\right)[p]_{\rho, q}^{p^{k-1}-1}\left[p^{k-1}-1\right]_{\rho^{p}, q^{p}} !
$$


If we put $0,1,2, \ldots, n$ instead of $k$, respectively, we then get

$$
\begin{aligned}
{\left[p^{0}-1\right]_{\rho, q} !=} & 1=(-1) \Gamma_{p}^{[\rho, q]}\left(p^{0}\right), \\
{\left[p^{1}-1\right]_{\rho, q} !=} & (-1)^{p} \Gamma_{p}^{[\rho, q]}\left(p^{1}\right)[p]_{\rho, q}^{p^{1-1}-1}\left[p^{1-1}-1\right]_{\rho^{p}, q^{p}} ! \\
& \vdots \\
{\left[p^{n}-1\right]_{\rho, q} !=} & (-1)^{p} \Gamma_{p}^{[\rho, q]}\left(p^{n}\right)[p]_{\rho, q}^{p^{n-1}-1}\left[p^{n-1}-1\right]_{\rho^{p}, q^{p}} !
\end{aligned}
$$

If we multiply to the both sides above, we attain

$$
\left[p^{n}-1\right]_{\rho, q} !=(-1)^{n p+1}[p]_{\rho, q}^{p^{0}+p^{1}+\cdots+p^{n-1}-n}\left[p^{n-1}-1\right]_{\rho^{p}, q^{p}} ! \prod_{j=0}^{n-2} \frac{\left[p^{j}-1\right]_{\rho^{p}, q^{p}} !}{\left[p^{j+1}-1\right]_{\rho, q^{\prime}} !} \prod_{j=0}^{n} \Gamma_{p}^{[\rho, q]}\left(p^{j}\right),
$$

which gives to the asserted result in Equation (18).

Theorem 7. For $n \in \mathbb{N}$, let $p$ be a prime number and $m_{n}$ be the sum of digits of $n=\sum_{j=0}^{m} a_{j} p^{j}\left(a_{m} \neq 0\right)$ in base $p$. The following identity holds true for $j=0,1, \ldots m$ :

$$
\frac{\left[\left\lfloor\frac{n}{p^{j}}\right\rfloor\right]_{\rho, q} !}{[p]_{\rho, q}^{\left\lfloor\frac{n}{p^{j}}\right\rfloor}\left[\left\lfloor\frac{n}{p^{j}}\right\rfloor\right]_{\rho^{p}, q^{p}} !}=\prod_{k=1}^{\left\lfloor\frac{n}{p^{j}}\right\rfloor} \frac{\rho^{k}-q^{k}}{\rho^{k p}-q^{k p}} \quad(0 \leqq k \leqq m) .
$$

Proof. For $0 \leqq j \leqq m$, we get

$$
\begin{aligned}
& \frac{\left[\left\lfloor\frac{n}{p^{j}}\right\rfloor\right]_{\rho, q} !}{[p]_{\rho, q}^{\left\lfloor\frac{n}{p^{j}}\right\rfloor}\left[\left\lfloor\frac{n}{p^{j}}\right\rfloor\right]_{\rho^{p}, q^{p}} !}=\frac{[1]_{\rho, q}[2]_{\rho, q} \cdots\left[\left\lfloor\frac{n}{p^{j}}\right\rfloor\right]_{\rho, q}}{[p]_{\rho, q}^{\left\lfloor\frac{n}{p^{j}}\right\rfloor}[1]_{\rho^{p}, q^{p}}[2]_{\rho^{p}, q^{p}} \cdots\left[\left\lfloor\frac{n}{p^{j}}\right\rfloor\right]_{\rho^{p}, q^{p}}}
\end{aligned}
$$

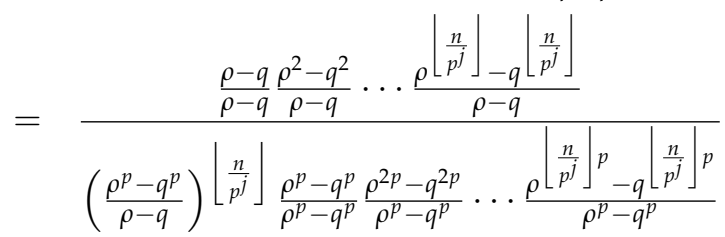

$$
\begin{aligned}
& =\frac{(\rho-q)\left(\rho^{2}-q^{2}\right) \cdots\left(\rho^{\left\lfloor\frac{n}{p^{j}}\right\rfloor}-q^{\left\lfloor\frac{n}{p^{j}}\right\rfloor}\right)}{\left(\rho^{p}-q^{p}\right)\left(\rho^{2 p}-q^{2 p}\right) \cdots\left(\rho^{\left\lfloor\frac{n}{p^{j}}\right\rfloor p}-q^{\left\lfloor\frac{n}{p^{j}}\right\rfloor p}\right)},
\end{aligned}
$$

which completes the proof of this theorem.

The following result can be easily derived from Theorems 5 and 7 .

Corollary 4. For $n \in N$, let $p$ be a prime number and $m_{n}$ be the sum of digits of $n=\sum_{j=0}^{m} a_{j} p^{j}\left(a_{m} \neq 0\right)$ in base $p$. We then get

$$
[n]_{\rho, q} !=(-1)^{\left(n-m_{n}\right) /(p-1)+n+1-m} \prod_{k=1}^{\left\lfloor\frac{n}{p^{1}}\right\rfloor} \frac{\left(\rho^{k p}-q^{k p}\right)}{\left(\rho^{k}-q^{k}\right)} \cdots \prod_{k=1}^{\left\lfloor\frac{n}{p^{m}}\right\rfloor} \frac{\left(\rho^{k p}-q^{k p}\right)}{\left(\rho^{k}-q^{k}\right)} \prod_{i=0}^{m} \Gamma_{p}^{[\rho, q]}\left(\left\lfloor\frac{n}{p^{i}}\right\rfloor+1\right) .
$$


We here provide a representation for $\Gamma_{p}^{[\rho, q]}(-n)$ via the following theorem.

Theorem 8. The following relation holds true for any prime $p$ and for any $n \in \mathbb{N}$ :

$$
\Gamma_{p}^{[\rho, q]}(-n)=(-1)^{n+1-\left\lfloor\frac{n}{p}\right\rfloor} \prod_{\substack{j<n+1 \\(p, j)=1}}(\rho q)^{j}\left(\Gamma_{p}^{[\rho, q]}(n+1)\right)^{-1} .
$$

Proof. In view of Lemma 1 and Theorem 3, we can write

$$
\begin{aligned}
1 & =\Gamma_{p}^{[\rho, q]}(0)=\Gamma_{p}^{[\rho, q]}(1+(-1))=\epsilon_{p}^{[\rho, q]}(-1) \Gamma_{p}^{[\rho, q]}(-1) \\
& =\epsilon_{p}^{[\rho, q]}(-1) \epsilon_{p}^{[\rho, q]}(-2) \Gamma_{p}^{[\rho, q]}(-2)=\cdots=\prod_{j=1}^{n} \epsilon_{p}^{[\rho, q]}(-j) \Gamma_{p}^{[\rho, q]}(-n),
\end{aligned}
$$

therefore, we get

$$
\left(\Gamma_{p}^{[\rho, q]}(-n)\right)^{-1}=\prod_{j=1}^{n} \epsilon_{p}^{[\rho, q]}(-j)
$$

By utilizing the definitons of $(\rho, q)$-numbers and $\epsilon_{p}^{[\rho, q]}$, we have

$$
\begin{aligned}
\left(\Gamma_{p}^{[\rho, q]}(-n)\right)^{-1} & =(-1)^{\left\lfloor\frac{n}{p}\right\rfloor} \prod_{\substack{j<n+1 \\
(p, j)=1}}(\rho q)^{-j}[j]_{\rho, q} \\
& =(-1)^{\left\lfloor\frac{n}{p}\right\rfloor-n-1} \prod_{\substack{j<n+1 \\
(p, j)=1}}(\rho q)^{-j}(-1)^{n+1} \prod_{\substack{j<n+1 \\
(p, j)=1}}[j]_{\rho, q} \\
& =(-1)^{\left\lfloor\frac{n}{p}\right\rfloor-n-1} \prod_{\substack{j<n+1 \\
(p, j)=1}}(\rho q)^{-j} \Gamma_{p}^{[\rho, q]}(n+1) .
\end{aligned}
$$

Thereby, the proof of this theorem is completed.

Corollary 5. Substituting $n-1$ by $n$ in Theorem 8 , one can readily write that

$$
\Gamma_{p}^{[\rho, q]}(n) \Gamma_{p}^{[\rho, q]}(1-n)=(-1)^{n-\left\lfloor\frac{n-1}{p}\right\rfloor} \prod_{\substack{j<n \\(p, j)=1}}(\rho q)^{j} .
$$

Now, we introduce $l: \mathbb{Z}_{p} \rightarrow\{1,2, \cdots, p\}$ by assigning to $x \in \mathbb{Z}_{p}$ its residue modulo $p \mathbb{Z}_{p}$. Let $n=a_{0}+a_{1} p+a_{2} p^{2}+\cdots$ be a positive in base $p$. If $a_{0} \neq 0$, then $\left\lfloor\frac{n-1}{p}\right\rfloor=a_{1}+a_{2} p+\cdots$. Thus, we obtain $n-p\left\lfloor\frac{n-1}{p}\right\rfloor=a_{0}=l(n)$. If $a_{0}=0$, then $n-1=p-1+b_{1} p+b_{2} p^{2}+\cdots$. Thus, $\left\lfloor\frac{n-1}{p}\right\rfloor=b_{1}+b_{2} p+\cdots$. Thus, we get $n-p\left\lfloor\frac{n-1}{p}\right\rfloor=1+(p-1)=p=l(n)$.

Hence, we give the following theorem.

Theorem 9. For $p \neq 2$ and all $x \in \mathbb{Z}_{p}$, we have

$$
\Gamma_{p}^{[\rho, q]}(x) \Gamma_{p}^{[\rho, q]}(1-x)=(-1)^{l(x)} \lim _{n \rightarrow x} \prod_{\substack{j<n \\(p, j)=1}}(\rho q)^{j} .
$$


Letting $x=\frac{1}{2}$ in Theorem 9 yields to the following result

$$
\begin{aligned}
& \left(\Gamma_{p}^{[\rho, q]}\left(\frac{1}{2}\right)\right)^{2}=(-1)^{l\left(\frac{1}{2}\right)} \lim _{n \rightarrow \frac{1}{2}} \prod_{\substack{j<n \\
(p, j)=1}}(\rho q)^{j} \\
& = \begin{cases}-\lim _{n \rightarrow \frac{1}{2}} \prod_{\substack{j<n \\
(p, j)=1}}(\rho q)^{j} & \text { if } p \equiv 1(\bmod 4), \\
\lim _{n \rightarrow \frac{1}{2}} \prod_{\substack{j<n \\
(p, j)=1}}(\rho q)^{j} & \text { if } p \equiv 3(\bmod 4),\end{cases}
\end{aligned}
$$

where we used the equality $l\left(\frac{1}{2}\right)=l\left(\frac{p+1}{2}\right)=\frac{p+1}{2}$ by definition.

Corollary 6. We have for $p=2$ in Theorem 8 ,

$$
\Gamma_{2}^{[\rho, q]}(n+1) \Gamma_{2}^{[\rho, q]}(-n)=(-1)^{n+1-\left\lfloor\frac{n}{2}\right\rfloor} \prod_{\substack{j<n+1 \\(2, j)=1}}(\rho q)^{j}=(-1)^{n+1-\left\lfloor\frac{n}{2}\right\rfloor}(\rho q)^{\left(n-\left\lfloor\frac{n}{2}\right\rfloor\right)^{2} .}
$$

We give an identity for special case $p=2$.

Theorem 10. For all $x \in \mathbb{Z}_{2}$, we obtain

$$
\Gamma_{2}^{[\rho, q]}(x) \Gamma_{2}^{[\rho, q]}(1-x)=(-1)^{1+\eta_{1}(x)} \lim _{n \rightarrow x} \prod_{\substack{j<n \\(2, j)=1}}(\rho q)^{j},
$$

where $\eta_{1}\left(\sum_{j=0}^{\infty} a_{j} 2^{j}\right)=a_{1}$.

Proof. For $n \in \mathbb{N}$, by Equation (20), we have

$$
\Gamma_{2}^{[\rho, q]}(n) \Gamma_{2}^{[\rho, q]}(1-n)=(-1)^{n-\left\lfloor\frac{n-1}{2}\right\rfloor} \prod_{\substack{j<n \\(2, j)=1}}(\rho q)^{j} .
$$

Let $n=a_{0}+a_{1} 2+a_{2} 2^{2}+\cdots$ in base 2. If $a_{0} \neq 0$, thereby $a_{0}=1$ in base 2 and $\left\lfloor\frac{n-1}{2}\right\rfloor=$ $a_{1}(\bmod 2)$. Hence, we obtain $(-1)^{n-\left\lfloor\frac{n-1}{2}\right\rfloor}=(-1)^{a_{0}-a_{1}}=(-1)^{1+a_{1}}=(-1)^{1+\eta_{1}(n)}$. If $a_{0}=0$, then we see $\left\lfloor\frac{n-1}{2}\right\rfloor=\left\lfloor\frac{-1+a_{1} 2+a_{2} 2^{2}+\cdots}{2}\right\rfloor=\left\lfloor\frac{1+\left(a_{1}-1\right) 2+a_{2} 2^{2}+\cdots}{2}\right\rfloor=a_{1}-1(\bmod 2)$. Therefore, we get $(-1)^{n-\left\lfloor\frac{n-1}{2}\right\rfloor}=(-1)^{2-\left(a_{1}-1\right)}=(-1)^{1+a_{1}}=(-1)^{1+\eta_{1}(n)}$. Consequently, we derive the following identity

$$
\Gamma_{2}^{[\rho, q]}(n) \Gamma_{2}^{[\rho, q]}(1-n)=(-1)^{1+\eta_{1}(n)} \prod_{\substack{j<n \\(2, j)=1}}(\rho q)^{j},
$$

which provides the claimed result in Equation (23).

\section{The $p$-adic $(\rho, q)$-Euler Constant}

The $p$-adic Euler constant $\gamma_{p} \in \mathbb{Q}_{p}$ is firstly given by Diamond [2] in 1977 as follows:

$$
\gamma_{p}=\frac{-\Gamma_{p}^{\prime}(1)}{\Gamma_{p}(1)} .
$$

In this section, we explore the $(\rho, q)$-analog of the $p$-adic Euler constant. We can readily consider that $\Gamma_{p}^{[\rho, q]}$ is locally analytic function thanks to Lemma 1. 
Then, we derive the following theorem.

Theorem 11. For $n \in \mathbb{N}$, we have

$$
\frac{\left(\Gamma_{p}^{[\rho, q]}\right)^{\prime}(n)}{\Gamma_{p}^{[\rho, q]}(n)}=\frac{\left(\Gamma_{p}^{[\rho, q]}\right)^{\prime}(1)}{\Gamma_{p}^{[\rho, q]}(1)}+\frac{1}{(\rho-q)} \sum_{j=1}^{n-1} \frac{\rho^{j} \log \rho-q^{j} \log q}{[j]_{\rho, q}} .
$$

Proof. From Theorem 3, we know that

$$
\log \left(\Gamma_{p}^{[\rho, q]}(n)\right)=\log \left(\Gamma_{p}^{[\rho, q]}(n-1)\right)+\log \left(\epsilon_{p}^{[\rho, q]}(n-1)\right) .
$$

Then,

$$
\begin{aligned}
\frac{\left(\Gamma_{p}^{[\rho, q]}\right)^{\prime}(n)}{\Gamma_{p}^{[\rho, q]}(n)} & =\frac{\left(\Gamma_{p}^{[\rho, q]}\right)^{\prime}(n-1)}{\Gamma_{p}^{[\rho, q]}(n-1)}+\frac{\left(\epsilon_{p}^{[\rho, q]}\right)^{\prime}(n-1)}{\epsilon_{p}^{[\rho, q]}(n-1)} \\
& =\frac{\left(\Gamma_{p}^{[\rho, q]}\right)^{\prime}(1)}{\Gamma_{p}^{[\rho, q]}(1)}+\sum_{j=1}^{n-1} \frac{\left(\epsilon_{p}^{[\rho, q]}\right)^{\prime}(j)}{\epsilon_{p}^{[\rho, q]}(j)},
\end{aligned}
$$

which implies the desired result in Equation (24).

Remark 3. Equation (24) can be called $(\rho, q)$-generalization of the known formula for p-adic gamma function

$$
\frac{\Gamma_{p}^{\prime}(n)}{\Gamma_{p}(n)}=\frac{\Gamma_{p}^{\prime}(1)}{\Gamma_{p}(1)}+\sum_{\substack{j<n \\(p, j)=1}} \frac{1}{j^{\prime}}
$$

or $(\rho, q)$-generalization of $p$-adic analog of the formula for classical gamma function

$$
\frac{\Gamma^{\prime}(n)}{\Gamma(n)}=\frac{\Gamma^{\prime}(1)}{\Gamma(1)}+\sum_{j<n} \frac{1}{j}
$$

Thereby, we are ready to define $(\rho, q)$-analog of the $p$-adic Euler constant $\gamma_{p}^{[\rho, q]}$ as follows

$$
\gamma_{p}^{[\rho, q]}:=\frac{\left(\Gamma_{p}^{[\rho, q]}\right)^{\prime}(1)}{\Gamma_{p}^{[\rho, q]}(1)}=\left(\Gamma_{p}^{[\rho, q]}\right)^{\prime}(1)=-\left(\Gamma_{p}^{[\rho, q]}\right)^{\prime}(0) .
$$

The $p$-adic $(\rho, q)$-Euler constant has a limit representation by the following theorem.

Theorem 12. We have

$$
\gamma_{p}^{[\rho, q]}=\lim _{n \rightarrow \infty} p^{-n}\left\{1-(-1)^{p} \frac{\left[p^{n}-1\right]_{\rho, q} !}{[p]_{\rho, q}^{p^{n-1}-1}\left[\left\lfloor\frac{n}{p}\right\rfloor\right]_{\rho^{p}, q^{p}} !}\right\} .
$$

Proof. In conjunction with Equation (15), we have

$$
\Gamma_{p}^{[\rho, q]}\left(p^{n}\right)=(-1)^{p} \frac{\left[p^{n}-1\right]_{\rho, q} !}{[p]_{\rho, q}^{p^{n-1}-1}\left[\left\lfloor\frac{n}{p}\right\rfloor\right]_{\rho^{p}, q^{p}} !} .
$$


Then, we investigate

$$
\begin{aligned}
\lim _{n \rightarrow \infty} p^{-n}\left\{1-(-1)^{p} \frac{\left[p^{n}-1\right]_{\rho, q} !}{[p]_{\rho, q}^{p^{n-1}-1}\left[\left\lfloor\frac{n}{p}\right\rfloor\right]_{\rho^{p}, q^{p}} !}\right\} & =\lim _{n \rightarrow \infty} \frac{1-\Gamma_{p}^{[\rho, q]}\left(p^{n}\right)}{p^{n}} \\
& =-\left(\Gamma_{p}^{[\rho, q]}\right)^{\prime}(0)=\gamma_{p}^{[\rho, q]} .
\end{aligned}
$$

Corollary 7. By means of the Lemma 1, we deduce that $\left|\gamma_{p}^{[\rho, q]}\right|_{p}=\left|\left(\Gamma_{p}^{[\rho, q]}\right)^{\prime}(1)\right|_{p} \leqq 1$.

\section{The $p$-adic $(\rho, q)$-Beta Function}

In this section, we define $(\rho, q)$-extension $p$-adic beta function by means of the $p$-adic $(\rho, q)$-gamma function discussed in Section 2. Then, we present several properties, identities and relations for the mentioned beta function.

The classical beta function $B(x, y)$ is defined by means of the classical gamma functions as follows:

$$
B(x, y)=\frac{\Gamma(x) \Gamma(y)}{\Gamma(x+y)}, \quad(x, y \in \mathbb{N})
$$

which also have the following subsequent properties (cf. [10]):

$$
\begin{gathered}
B(x, y)=B(y, x) \\
B(x, y)=B(x, y+1)+B(x+1, y) \\
B(x+1, y)=B(x, y) \frac{x}{x+y} \\
B(x, y+1)=B(x, y) \frac{y}{x+y} \\
B(x+1, y)=\frac{x}{y} B(x, y+1) .
\end{gathered}
$$

The $p$-adic beta function is defined by means of the $p$-adic gamma functions as follows:

$$
B_{p}(x, y)=\frac{\Gamma_{p}(x) \Gamma_{p}(y)}{\Gamma_{p}(x+y)}, \quad\left(x, y \in \mathbb{Z}_{p}\right)
$$

which also have the following subsequent properties (cf. $[5,10])$ :

$$
\begin{gathered}
B_{p}(x, y)=B_{p}(y, x) \\
B_{p}(x, y)=\frac{h_{p}(x+y)}{h_{p}(x)+h_{p}(y)}\left(B_{p}(x, y+1)+B_{p}(x+1, y)\right) \\
B_{p}(x+1, y)=B_{p}(x, y) \frac{x}{x+y} \\
B_{p}(x, y+1)=B_{p}(x, y) \frac{y}{x+y} \\
B_{p}(x+1, y)=\frac{h_{p}(x)}{h_{p}(y)} B_{p}(x, y+1) .
\end{gathered}
$$


Definition 3. Let $\rho$ and $q \in \mathbb{C}_{p}$ with $|\rho-1|_{p}<1$ and $|q-1|_{p}<1, \rho \neq 1$ and $q \neq 1$. We define the $p$-adic $(\rho, q)$-beta function via the $p$-adic $(\rho, q)$-gamma functions as follows:

$$
B_{p}^{[\rho, q]}(x, y)=\frac{\Gamma_{p}^{[\rho, q]}(x) \Gamma_{p}^{[\rho, q]}(y)}{\Gamma_{p}^{[\rho, q]}(x+y)},
$$

for $x, y \in \mathbb{Z}_{p}$.

Remark 4. In the case $\rho=1$, the $p$-adic $(\rho, q)$-beta function reduces to the the $p$-adic $q$-beta function (cf. [5]).

Remark 5. When $q \rightarrow \rho=1$, the $p$-adic $(\rho, q)$-beta function reduces to the usual $p$-adic beta function (cf. [10]).

We are now ready to investigate the properties of the $p$-adic $(\rho, q)$-beta function.

Theorem 13. For $x, y \in \mathbb{Z}_{p}$, the $p$-adic $(\rho, q)$-beta function is symmetric about $x$ and $y$ :

$$
B_{p}^{[\rho, q]}(x, y)=B_{p}^{[\rho, q]}(y, x) .
$$

Proof. By Equation (26), we readily get

$$
B_{p}^{[\rho, q]}(x, y)=\frac{\Gamma_{p}^{[\rho, q]}(x) \Gamma_{p}^{[\rho, q]}(y)}{\Gamma_{p}^{[\rho, q]}(x+y)}=\frac{\Gamma_{p}^{[\rho, q]}(y) \Gamma_{p}^{[\rho, q]}(x)}{\Gamma_{p}^{[\rho, q]}(y+x)}=B_{p}^{[\rho, q]}(x, y),
$$

which is the asserted result in Equation (27).

Theorem 14. For $x, y \in \mathbb{Z}_{p}$, the $p$-adic $(\rho, q)$-beta function has the following formula:

$$
B_{p}^{[\rho, q]}(x+1, y)=\frac{\epsilon_{p}^{[\rho, q]}(x)}{\epsilon_{p}^{[\rho, q]}(x+y)} B_{p}^{[\rho, q]}(x, y) .
$$

Proof. In view of Equations (12) and (26), we readily get

$$
\begin{aligned}
B_{p}^{[\rho, q]}(x+1, y) & =\frac{\Gamma_{p}^{[\rho, q]}(x+1) \Gamma_{p}^{[\rho, q]}(y)}{\Gamma_{p}^{[\rho, q]}(x+y+1)} \\
& =\frac{\epsilon_{p}^{[\rho, q]}(x) \Gamma_{p}^{[\rho, q]}(x) \Gamma_{p}^{[\rho, q]}(y)}{\epsilon_{p}^{[\rho, q]}(x+y) \Gamma_{p}^{[\rho, q]}(x+y)} \\
& =\frac{\epsilon_{p}^{[\rho, q]}(x)}{\epsilon_{p}^{[\rho, q]}(x+y)} \frac{\Gamma_{p}^{[\rho, q]}(x) \Gamma_{p}^{[\rho, q]}(y)}{\Gamma_{p}^{[\rho, q]}(x+y)}=\frac{\epsilon_{p}^{[\rho, q]}(x)}{\epsilon_{p}^{[\rho, q]}(x+y)} B_{p}^{[\rho, q]}(x, y),
\end{aligned}
$$

which is the desired result in Equation (28).

Theorem 15. For $x, y \in \mathbb{Z}_{p}$, the $p$-adic $(\rho, q)$-beta function satisfies the following identity:

$$
B_{p}^{[\rho, q]}(x, y+1)=\frac{\epsilon_{p}^{[\rho, q]}(y)}{\epsilon_{p}^{[\rho, q]}(x+y)} B_{p}^{[\rho, q]}(x, y) .
$$


Proof. By Equation (26), we readily get

$$
\begin{aligned}
B_{p}^{[\rho, q]}(x, y+1) & =\frac{\Gamma_{p}^{[\rho, q]}(x) \Gamma_{p}^{[\rho, q]}(y+1)}{\Gamma_{p}^{[\rho, q]}(x+y+1)} \\
& =\frac{\Gamma_{p}^{[\rho, q]}(x) \epsilon_{p}^{[\rho, q]}(y) \Gamma_{p}^{[\rho, q]}(y)}{\epsilon_{p}^{[\rho, q]}(x+y) \Gamma_{p}^{[\rho, q]}(x+y)} \\
& =\frac{\epsilon_{p}^{[\rho, q]}(y)}{\epsilon_{p}^{[\rho, q]}(x+y)} \frac{\Gamma_{p}^{[\rho, q]}(x) \Gamma_{p}^{[\rho, q]}(y)}{\Gamma_{p}^{[\rho, q]}(x+y)}=\frac{\epsilon_{p}^{[\rho, q]}(y)}{\epsilon_{p}^{[\rho, q]}(x+y)} B_{p}^{[\rho, q]}(x, y),
\end{aligned}
$$

which is the claimed result in Equation (29).

By Theorems 14 and 15, we see that

$$
\begin{aligned}
B_{p}^{[\rho, q]}(x+1, y)+B_{p}^{[\rho, q]}(x, y+1) & =\frac{\epsilon_{p}^{[\rho, q]}(x)}{\epsilon_{p}^{[\rho, q]}(x+y)} B_{p}^{[\rho, q]}(x, y)+\frac{\epsilon_{p}^{[\rho, q]}(y)}{\epsilon_{p}^{[\rho, q]}(x+y)} B_{p}^{[\rho, q]}(x, y) \\
& =\frac{\epsilon_{p}^{[\rho, q]}(x)+\epsilon_{p}^{[\rho, q]}(y)}{\epsilon_{p}^{[\rho, q]}(x+y)} B_{p}^{[\rho, q]}(x, y)
\end{aligned}
$$

and

$$
\begin{aligned}
B_{p}^{[\rho, q]}(x+1, y) & =\frac{\epsilon_{p}^{[\rho, q]}(x)}{\epsilon_{p}^{[\rho, q]}(x+y)} B_{p}^{[\rho, q]}(x, y) \\
& =\frac{\epsilon_{p}^{[\rho, q]}(x)}{\epsilon_{p}^{[\rho, q]}(y)} \frac{\epsilon_{p}^{[\rho, q]}(y)}{\epsilon_{p}^{[\rho, q]}(x+y)} B_{p}^{[\rho, q]}(x, y) \\
& =\frac{\epsilon_{p}^{[\rho, q]}(x)}{\epsilon_{p}^{[\rho, q]}(y)} B_{p}^{[\rho, q]}(x, y+1)
\end{aligned}
$$

which implies the following results.

Corollary 8. For $x, y \in \mathbb{Z}_{p}$, the following formulas are valid:

$$
B_{p}^{[\rho, q]}(x, y)=\frac{\epsilon_{p}^{[\rho, q]}(x+y)}{\epsilon_{p}^{[\rho, q]}(x)+\epsilon_{p}^{[\rho, q]}(y)}\left(B_{p}^{[\rho, q]}(x+1, y)+B_{p}^{[\rho, q]}(x, y+1)\right)
$$

and

$$
B_{p}^{[\rho, q]}(x+1, y)=\frac{\epsilon_{p}^{[\rho, q]}(x)}{\epsilon_{p}^{[\rho, q]}(y)} B_{p}^{[\rho, q]}(x, y+1) .
$$

We give the following theorem.

Theorem 16. Let $x, y \in \mathbb{Z}_{p}$. For $p=2$, we get

$$
B_{p}^{[\rho, q]}(x, y) B_{p}^{[\rho, q]}(x+y, 1-y)=\frac{(-1)^{1+\eta_{1}(x)}}{\epsilon_{p}^{[\rho, q]}(x)} \lim _{n \rightarrow y} \prod_{\substack{j<n \\(p, j)=1}}(\rho q)^{j}
$$


and for $p \neq 2$, we have

$$
B_{p}^{[\rho, q]}(x, y) B_{p}^{[\rho, q]}(x+y, 1-y)=\frac{(-1)^{l(y)}}{\epsilon_{p}^{[\rho, q]}(x)} \lim _{n \rightarrow y} \prod_{\substack{j<n \\(p, j)=1}}(\rho q)^{j} .
$$

Proof. From Definition 3, we easily compute that

$$
\begin{aligned}
B_{p}^{[\rho, q]}(x, y) B_{p}^{[\rho, q]}(x+y, 1-y) & =\frac{\Gamma_{p}^{[\rho, q]}(x) \Gamma_{p}^{[\rho, q]}(y)}{\Gamma_{p}^{[\rho, q]}(x+y)} \frac{\Gamma_{p}^{[\rho, q]}(x+y) \Gamma_{p}^{[\rho, q]}(1-y)}{\Gamma_{p}^{[\rho, q]}(x+1)} \\
& =\frac{\Gamma_{p}^{[\rho, q]}(x) \Gamma_{p}^{[\rho, q]}(y) \Gamma_{p}^{[\rho, q]}(1-y)}{\epsilon_{p}^{[\rho, q]}(x) \Gamma_{p}^{[\rho, q]}(x)} \\
& =\frac{\Gamma_{p}^{[\rho, q]}(y) \Gamma_{p}^{[\rho, q]}(1-y)}{\epsilon_{p}^{[\rho, q]}(x)} .
\end{aligned}
$$

It just remains to use Equations (21) and (23) to obtain desired result in Equations (30) and (31).

We provide the following theorem.

Theorem 17. Let $x, y \in \mathbb{Z}_{p}$. We then obtain

$$
B_{p}^{[\rho, q]}(x+1, y+1)=\frac{\epsilon_{p}^{[\rho, q]}(x) \epsilon_{p}^{[\rho, q]}(y)}{\epsilon_{p}^{[\rho, q]}(x+y+1) \epsilon_{p}^{[\rho, q]}(x+y)} B_{p}^{[\rho, q]}(x, y) .
$$

Proof. In view of Definition 3 and using Equation (12), we readily see that

$$
\begin{aligned}
B_{p}^{[\rho, q]}(x+1, y+1) & =\frac{\Gamma_{p}^{[\rho, q]}(x+1) \Gamma_{p}^{[\rho, q]}(y+1)}{\Gamma_{p}^{[\rho, q]}(x+1+y+1)} \\
& =\frac{\Gamma_{p}^{[\rho, q]}(x+1) \epsilon_{p}^{[\rho, q]}(y) \Gamma_{p}^{[\rho, q]}(y)}{\epsilon_{p}^{[\rho, q]}(x+y+1) \Gamma_{p}^{[\rho, q]}(x+y+1)} \\
& =\frac{\epsilon_{p}^{[\rho, q]}(y)}{\epsilon_{p}^{[\rho, q]}(x+y+1)} B_{p}^{[\rho, q]}(x+1, y),
\end{aligned}
$$

which implies the asserted Equation (32) thanks to Equation (28).

For $x, y, \xi, \gamma \in \mathbb{Z}_{p}$, we note that

$$
B_{p}^{[\rho, q]}(x, y) B_{p}^{[\rho, q]}(x+y, \xi) B_{p}^{[\rho, q]}(x+y+\xi, \gamma)=\frac{\Gamma_{p}^{[\rho, q]}(x) \Gamma_{p}^{[\rho, q]}(y) \Gamma_{p}^{[\rho, q]}(\xi) \Gamma_{p}^{[\rho, q]}(\gamma)}{\Gamma_{p}^{[\rho, q]}(x+y+\xi+\gamma)} .
$$

We give the following theorem.

Theorem 18. Let $x \in \mathbb{Z}_{p}$. For $p=2$, we obtain

$$
B_{p}^{[\rho, q]}(x, 1-x)=(-1)^{2+\eta_{1}(x)} \lim _{n \rightarrow y} \prod_{\substack{j<n \\(p, j)=1}}(\rho q)^{j}
$$


and for $p \neq 2$, we attain

$$
B_{p}^{[\rho, q]}(x, 1-x)=(-1)^{l(y)+1} \lim _{n \rightarrow y} \prod_{\substack{j<n \\(p, j)=1}}(\rho q)^{j}
$$

Proof. From Definition 3, we easily compute that

$$
B_{p}^{[\rho, q]}(x, 1-x)=\frac{\Gamma_{p}^{[\rho, q]}(x) \Gamma_{p}^{[\rho, q]}(1-x)}{\Gamma_{p}^{[\rho, q]}(1)}=-\Gamma_{p}^{[\rho, q]}(x) \Gamma_{p}^{[\rho, q]}(1-x),
$$

which implies the claimed result in Equations (33) and (34) in conjunction with Equations (21) and (23).

By the motivation for usual binomial coefficient, for $n, k \in \mathbb{N}$ with $n \geq k$, we consider the $p$-adic $(\rho, q)$-binomial coefficients $\left(\begin{array}{l}n \\ k\end{array}\right) \begin{aligned} & {[\rho, q]} \\ & p\end{aligned}$ by means of the $p$-adic $(\rho, q)$-factorial in Equation (6) as follows:

$$
\left(\begin{array}{l}
n \\
k
\end{array}\right)_{p}^{[\rho, q]}=\frac{(n !)_{p}^{[\rho, q]}((n-k) !)_{p}^{[\rho, q]}}{(k !)_{p}^{[\rho, q]}}
$$

Thus, we give the following theorem.

Theorem 19. Let $n, k \in \mathbb{N}$ with $n \geq k$. We have

$$
\left(\begin{array}{l}
n \\
k
\end{array}\right)_{p}^{[\rho, q]} B_{p}^{[\rho, q]}(n-k+1, k+1)=-\frac{1}{\epsilon_{p}^{[\rho, q]}(n+1)}
$$

Proof. The proof just follows from Equations (26) and (35) with Equation (12).

We provide the following theorem.

Theorem 20. Let $n, k \in \mathbb{N}$. We have

$$
B_{p}^{[\rho, q]}(-n,-k)=(-1)^{1+\left\lfloor\frac{n+k}{p}\right\rfloor-\left\lfloor\frac{n}{p}\right\rfloor-\left\lfloor\frac{k}{p}\right\rfloor} \frac{\epsilon_{p}^{[\rho, q]}(n+k)}{\epsilon_{p}^{[\rho, q]}(n) \epsilon_{p}^{[\rho, q]}(k)} \frac{1}{B_{p}^{[\rho, q]}(n, k)} \frac{\prod_{\substack{j<k+1 \\(p, j)=1}}(\rho q)^{j}}{\prod_{\substack{j<n+1 \\(p, j)=1}}(\rho q)^{j}} .
$$

Proof. The proof of this theorem just follows from Equations (26) and (12) and Theorem 8 with some basic computations.

Finally, we present the following theorem.

Theorem 21. Let $x \in \mathbb{Z}_{p}$. For $p=2$, we obtain

$$
B_{p}^{[\rho, q]}(x, 1-x)=(-1)^{2+\eta_{1}(x)} \lim _{n \rightarrow y} \prod_{\substack{j<n \\(p, j)=1}} q^{j}
$$

and for $p \neq 2$, we attain

$$
B_{p}^{[\rho, q]}(x, 1-x)=(-1)^{l(y)+1} \lim _{n \rightarrow y} \prod_{\substack{j<n \\(p, j)=1}} q^{j}
$$


Proof. From Definition 3, we easily compute that

$$
B_{p}^{[\rho, q]}(x, 1-x)=\frac{\Gamma_{p}^{[\rho, q]}(x) \Gamma_{p}^{[\rho, q]}(1-x)}{\Gamma_{p}^{[\rho, q]}(1)}=-\Gamma_{p}^{[\rho, q]}(x) \Gamma_{p}^{[\rho, q]}(1-x),
$$

which implies the claimed result in Equations (33) and (34) in conjunction with Equations (21) and (23).

Remark 6. The results derived in this part are generalizations of the results obtained in $[5,10]$.

\section{Conclusions}

In this paper, we have firstly generalized $p$-adic factorial function and $p$-adic gamma function based on $(\rho, q)$-numbers. Utilizing these generalizations, we have constructed some recurrence relations and identities. By using some properties of $(\rho, q)$-numbers, we have derived several new and interesting identities and formulas for $(n !)_{p}^{[\rho, q]}$ and $\Gamma_{p}^{[\rho, q]}(x)$. As an application, we have derived the $p$-adic $(\rho, q)$-Euler constant by means of the $p$-adic $(\rho, q)$-gamma function and have given a limit representation for the foregoing constant. Moreover, we have considered $(\rho, q)$-extension of the $p$-adic beta function via the $p$-adic $(\rho, q)$-gamma function and then we have acquired several formulas and identities.

Author Contributions: U.D. wrote the most part of this paper. M.A. took part in deriving some relations and editing this paper. Authors together discussed some applications of these functions considered in this paper.

Conflicts of Interest: The authors declare no conflict of interest.

\section{References}

1. Schikhof, W.H. Ultrametric Calculus: An Introduction to p-adic Analysis; Cambridge University Press: Cambridge, UK, 1984.

2. Diamond, J. The $p$-adic log gamma function and $p$-adic Euler constant. Trans. Am. Math. Soc. 1977, 233, 321-337.

3. Havara, Ö.Ç.; Menken, H. On the Volkenborn integral of the $q$-extension of the $p$-adic gamma function. J. Math. Anal. 2017, 8, 64-72.

4. Havara, Ö.Ç.; Menken, H. Some properties of the p-adic log gamma function. J. Math. Anal. 2016, 7, 26-32.

5. Havara, Ö.Ç.; Menken, H. Some properties of the $q$-extension of the $p$-adic beta function. Neural Parallel Sci. Comput. 2014, 24, 409-418.

6. Kim, Y.S. $q$-analogues of $p$-adic gamma functions and p-adic Euler constants. Commun. Korean Math. Soc. 1998, 13, 735-741.

7. Koblitz, N. p-adic Numbers, p-adic Analysis, and Zeta Functions; Springer: New York, NY, USA, 1977.

8. Koblitz, N. q-extension of the $p$-adic gamma function. Trans. Am. Math. Soc. 1980, 260, 449-457.

9. Menken, H.; Körükçü, A. Some properties of the $q$-extension of the $p$-adic gamma function. Abstr. Appl. Anal. 2013, 2013, 176470. [CrossRef]

10. Menken, H.; Çolakoğlu, Ö. Some properties of the p-adic beta function. Eur. J. Pure Appl. Math. 2015, 8, 214-231.

11. Morita, Y. A p-adic analogue of the Г-function. J. Fac. Sci. 1975, 22, 255-266.

12. Nakazako, H. The $q$-analogue of the $p$-adic gamma function. Kodai Math. J. 1988, 11, 141-153. [CrossRef]

13. Robert, A.M. A Course in p-adic Analysis; Springer: New York, NY, USA, 2000.

14. Acikgoz, M.; Aslan, N.; Köskeroğlu, N.; Araci, S. p-adic approach to linear 2-normed spaces. Math. Morav. 2009, 13, 7-22. [CrossRef]

15. Araci, S.; Duran, U.; Acikgoz, M. $(\rho, q)$-Volkenborn integration. J. Number Theory 2017, 171, 18-30. [CrossRef]

16. Aral, A.; Gupta, V. Application of $(p, q)$-gamma function to Szász Durrmeyer operator. Publications de l'Institut Mathematique 2017, 102, 211-220. [CrossRef] 
17. Duran, U.; Acikgoz, M. On $(\rho, q)$-Euler numbers and polynomials associated with $(\rho, q)$-Volkenborn integrals. Int. J. Number Theory 2018, 14, 241-253. [CrossRef]

18. Sadjang, P.N. On the $(p, q)$-gamma and the $(p, q)$-beta functions. arXiv 2015, arXiv:1506.07394.

19. Sadjang, P.N. On the fundamental theorem of $(p, q)$-calculus and some $(p, q)$-Taylor formulas. arXiv 2013, arXiv:1309.3934v1.

20. Sahai, V.; Yadav, S. Representations of two parameter quantum algebras and $p, q$-special functions. J. Math. Anal. Appl. 2007, 335, 268-279. [CrossRef]

21. Koblitz, N. On Carlitz's $q$-Bernoulli numbers. J. Number Theory 1982, 14, 332-339. [CrossRef]

22. Kim, M.-S.; Son, J.W. Analytic properties of the $q$-Volkenborn integral on the ring of $p$-adic integers. Bull. Korean Math. Soc. 2007, 44, 1-12. [CrossRef]

23. Artin, E. The Gamma Function; Holt, Rinehart and Winston: New York, NY, USA, 1964.

24. Chakrabarti, R.; Jagannathan, R. A $(p, q)$-oscillator realization of two-parameter quantum algebras. J. Phys. A Math. Gen. 1991, 24, L711. [CrossRef]

25. Milovanovic, G.V.; Gupta, V.; Malik, N. $(p, q)$-Beta functions and applications in approximation. Bol. Soc. Mat. Mex. 2016. [CrossRef]

(C) 2019 by the authors. Licensee MDPI, Basel, Switzerland. This article is an open access article distributed under the terms and conditions of the Creative Commons Attribution (CC BY) license (http://creativecommons.org/licenses/by/4.0/). 